Real Analysis Exchange

Vol. 26(1), 2000/2001, pp. 467-470

Guanzhen Zhou, Department of Mathematics, Ningbo University, Ningbo, Zhejiang 315211 China and Department of Mathematics,Zhejiang University, Hangzhou, Zhejiang 310028 China.

Songping Zhou, The Mathematical Institute, Ningbo University, Ningbo, Zhejiang 315211 China. e-mail: spz@mail.hz.zj.cn

\title{
ON SERIES OF NON-NEGATIVE TERMS
}

\author{
Abstract \\ If $\sum a_{k}$ and $\sum b_{k}$ are series of non-negative terms, we provide a \\ necessary and sufficient condition that $\sup _{n}\left\{\sum_{1}^{n} a_{k} / \sum_{1}^{n} b_{k}\right\}=\infty$.
}

The properties of infinite series all of whose terms are greater than or equal to 0 , are of importance in many problems of analysis. Given two such series, $\sum a_{k}$ and $\sum b_{k}$, we shall be interested in the magnitude of the ratio of the partial sums. We give two examples of problems in which this ratio figures.

Let $\Lambda=\left\{\lambda_{k}\right\}$ be an increasing sequence of positive numbers such that $\sum 1 / \lambda_{k}=\infty$. If $f$ is a real function of a real variable and $\left\{I_{k}\right\}=\left\{\left[a_{k}, b_{k}\right]\right\}$ is a collection of nonoverlapping intervals in its domain, we write $f\left(I_{k}\right)=$ $f\left(b_{k}\right)-f\left(a_{k}\right)$. The class of functions for which $\sup _{\left\{I_{n}\right\}} \sum\left|f\left(I_{k}\right)\right| / \lambda_{k}<\infty$ are known as the functions of $\Lambda$-bounded variation, often referred to as the Waterman class $\Lambda B V$ ([1], for an overview see [2]). If $\Lambda$ is as above and $\Gamma=\left\{\gamma_{k}\right\}$ is another such sequence, then Perlman and Waterman [3] showed that $\Lambda B V \supsetneq \Gamma B V$ if and only if

$$
\sup _{n} \frac{\sum_{1}^{n} \frac{1}{\gamma_{k}}}{\sum_{1}^{n} \frac{1}{\lambda_{k}}}=\infty .
$$

More recently, Kvernadze[4], in studying Lagrange interpolation, showed that if $\left\{\lambda_{k}\right\}$ is a nondecreasing sequence satisfying

$$
\sup _{n} \frac{\sum_{1}^{n} k^{q-1 / 2}}{\sum_{1}^{n} \frac{1}{\lambda_{k}}}=\infty,
$$

Key Words: positive series, generalized bounded variation, Waterman classes

Mathematical Reviews subject classification: Primary 40A05; Secondary 26A45

Received by the editors May 29, 2000

* Supported in part by Ningbo Key Doctoral Funds and Zhejiang National Science Foundation of China 
where $q>-1 / 2$, then there is a sequence $\left\{\varepsilon_{k}\right\} \searrow 0$ such that

$$
\sum \frac{\varepsilon_{k}}{\lambda_{k}}<\infty \text { and } \sum \frac{\varepsilon_{k}}{k^{q-1 / 2}}=\infty .
$$

Here we shall give a necessary and sufficient condition for

$$
\sup _{n}\left\{\frac{\sum_{1}^{n} a_{k}}{\sum_{1}^{n} b_{k}}\right\}=\infty
$$

for arbitrary series of non-negative terms. Note in particular that we do not assume $\left\{a_{k}\right\}$ and $\left\{b_{k}\right\}$ to be monotone.

Theorem 1. Let $\left\{a_{k}\right\}$ and $\left\{b_{k}\right\}$ be two sequences of non-negative numbers, $b_{1} \neq 0$. Then

$$
\sup _{n} \sum_{1}^{n} a_{k} / \sum_{1}^{n} b_{k}=\infty
$$

if and only if there exists a monotone-decreasing null sequence $\left\{\varepsilon_{k}\right\}$ such that

$$
\sum_{1}^{n} \varepsilon_{k} b_{k}<\infty \text { and } \sum_{1}^{n} \varepsilon_{k} a_{k}=\infty
$$

Proof. If (1) holds, then there is a subsequence of the natural numbers $\left\{n_{i}\right\}$ such that

$$
\sum_{1}^{n_{i}} a_{k}>2^{i} \sum_{1}^{n_{i}} b_{k}
$$

It is clear that (1) implies $\sum_{1}^{\infty} a_{k}=\infty$, so there is a subsequence $\left\{n_{i_{j}}\right\}$ such that

$$
\sum_{1}^{n_{i_{j+1}}} a_{k}>2 \sum_{1}^{n_{i_{j}}} a_{k}
$$

From (2) and (3) we have

$$
\sum_{n_{i_{j}+1}}^{n_{i_{j+1}}} a_{k} \geqslant \frac{1}{2} \sum_{1}^{n_{i_{j+1}}} a_{k} \geqslant 2^{i_{j+1}-1} \sum_{1}^{n_{i_{j+1}}} b_{k} .
$$

For $n_{i_{j}+1} \leqslant k \leqslant n_{i_{j+1}}$, let

$$
\varepsilon_{k}=\frac{1}{2^{i_{j+1}} \sum_{l=1}^{n_{i_{j}+1}} b_{l}}
$$


and, for $k=1,2, \ldots, n_{i_{1}}$, let $\varepsilon_{k}$ be chosen so that $\left\{\varepsilon_{k}\right\}_{1}^{\infty}$ is monotone. Thus $\left\{\varepsilon_{k}\right\}_{1}^{\infty}$ is a monotone-decreasing null sequence. From (4) and (5) we have

$$
\sum_{n_{i_{1}+1}}^{\infty} \varepsilon_{k} a_{k}=\sum_{j=1}^{\infty} \sum_{n_{i_{j}+1}}^{n_{i_{j+1}}} \varepsilon_{k} a_{k}=\sum_{j=1}^{\infty} \frac{1}{2^{i_{j+1}} \sum_{1}^{n_{i_{j+1}}} b_{k}} \sum_{n_{i_{j}+1}}^{n_{i_{j+1}}} a_{k} \geqslant \sum_{1}^{\infty} \frac{1}{2}=\infty
$$

and, at the same time,

$$
\sum_{n_{i_{1}+1}}^{\infty} \varepsilon_{k} b_{k}=\sum_{j=1}^{\infty} \frac{\sum_{n_{i_{j}+1}}^{n_{i_{j}+1}} b_{k}}{2^{i_{j+1}} \sum_{1}^{n_{i_{j}+1}} b_{k}} \leqslant \sum_{j=1}^{\infty} \frac{1}{2^{i_{j+1}}}<\infty
$$

which establishes the necessity of our condition.

Now we assume that (1) is false. Then there is an $M>0$ such that, for all $n$,

$$
\sum_{1}^{n} a_{k} \leqslant M \sum_{1}^{n} b_{k}
$$

Letting $A_{k}=\sum_{1}^{n} a_{j}, B_{k}=\sum_{1}^{n} b_{j}$ and applying Abel's partial summation, we have

$$
\begin{aligned}
\sum_{1}^{n} \varepsilon_{k} a_{k} & =\sum_{1}^{n-1} A_{k}\left(\varepsilon_{k}-\varepsilon_{k+1}\right)+A_{n} \varepsilon_{n} \\
& \leqslant M \sum_{1}^{n-1} B_{k}\left(\varepsilon_{k}-\varepsilon_{k+1}\right)+M B_{n} \varepsilon_{n}=M \sum_{1}^{n} \varepsilon_{k} b_{k}
\end{aligned}
$$

noting that $\varepsilon_{k}-\varepsilon_{k+1} \geqslant 0$ for all $k$. Thus we see that, under the assumption that (1) is false, $\sum \varepsilon_{k} a_{k}$ converges if $\sum \varepsilon_{k} b_{k}$ converges, which completes the proof.

Note that monotonicity of $\left\{a_{k}\right\}$ and $\left\{b_{k}\right\}$ was not required, unlike the particular case considered in [4].

Remark. In the proof of our theorem, the monotonicity of $\left\{\varepsilon_{k}\right\}$ was used strongly. In fact, it is essential to the validity of the theorem. Let us suppose

$$
a_{k}=\left\{\begin{array}{ll}
\frac{1}{k+1}, & k \text { even } \\
0, & k \text { odd }
\end{array} \quad b_{k}= \begin{cases}\frac{1}{k}, & k \text { odd } \\
0, & k \text { even }\end{cases}\right.
$$

and

$$
\varepsilon_{k}=\left\{\begin{array}{ll}
1 / \log k, & k \text { even } \\
1 / \log ^{2} k, & k \text { odd }
\end{array} .\right.
$$


Then $\left\{\varepsilon_{k}\right\}$ is a positive null sequence with $\sum \varepsilon_{k} a_{k}=\infty$ and $\sum \varepsilon_{k} b_{k}<\infty$, but

$$
\sup _{n} \sum_{1}^{n} a_{k} / \sum_{1}^{n} b_{k}=1 .
$$

\section{References}

[1] D. Waterman, On convergence of Fourier series of functions of generalized bounded variation, Studia Math. 44(1972), 107-117.

[2] M. Avdispahic, Concepts of generalized bounded variation and the theory of Fourier series, Internat. J. Math. Sci. 9(1986), 223-244.

[3] S. Perlman, D. Waterman, Some remarks on functions of $\Lambda$-bounded variation, Proc. Amer. Math. Soc. 74(1979), 113-118.

[4] G. Kvernadze, Uniform convergence of Lagrange interpolation based on the Jacobi nodes, J. Approx. Theory 87(1996), 179-193. 